\section{O parecer do Conselho Federal de Medicina, o incentivo à remuneração ao parto e as taxas de cesariana no Brasil}

\author{
The position of the Brazilian Federal Board of \\ Medicine on incentives for reimbursement of \\ childbirth care and the impact on cesarean rates
}

\author{
La opinión del Consejo Federal de Medicina, \\ remuneración por nacimiento y tasas \\ de cesárea en Brasil
}

Paulo Fontoura Freitas 1,2 Bianca Carvalho Moreira 2 Andre Luciano Manoel 2 Ana Clara de Albuquerque Botura ${ }^{2}$

\footnotetext{
${ }^{1}$ Centro de Ciências da Saúde, Universidade Federal de Santa Catarina, Florianópolis, Brasil.

2 Universidade do Sul de Santa Catarina, Tubarão, Brasil.

Correspondência P. F. Freitas

Centro de Ciências da Saúde Universidade Federal de Santa Catarina.

Campus Universitario Trindade, Florianópolis, SC 88062-490, Brasil. pfreitas.epidemio@gmail.com
}

\begin{abstract}
This study analyzed incentives for reimbursement of childbirth care advocated by the Brazilian Federal Board of Medicine (CFM) and their impact on cesarean rates. A consecutive sample of 600 postpartum women was surveyed. The overall cesarean rate was $59.2 \%$, as compared to 92.3\% among women that had the same physician for their prenatal care and childbirth. Cesarean rates were significantly greater in the groups of women with higher prevalence of the same physician during prenatal care and delivery, that is, higher rates were associated with older maternal age $(P R=1.65)$, more schooling $(P R=1.25)$, prenatal care in the private sector $(P R=1.39)$ or through private health plans $(P R$ $=1.43)$, previous cesarean section $(P R=2.78)$, and admission earlier in labor $(P R=1.93)$. The results challenge the position by the CFM that financial incentives for women to have the same obstetrician during prenatal care and labor would encourage normal childbirth, when these women are precisely the ones with the highest cesarean rates.
\end{abstract}

Parturition; Cesarean Section; Remuneration; Health Policy

\section{Resumo}

O estudo buscou investigar como o incentivo à remuneração ao parto, preconizado pelo Conselho Federal de Medicina (CFM) do Brasil, influencia as taxas de cesariana. Foi investigada uma amostra consecutiva de 600 puérperas. A proporção de cesarianas na amostra foi de 59,2\%, sendo 92,3\% entre as mulheres tendo parto e pré-natal com o mesmo profissional. As taxas de cesariana foram significativamente mais elevadas exatamente naqueles grupos com maior prevalência do mesmo profissional, ou seja, idade mais avançada $(R P=1,65)$, maior escolaridade $(R P=1,25)$, pré-natal privado $(R P=1,39)$ ou por convênio $(R P=1,43)$, cesariana prévia $(R P=2,78)$ e admitidas precocemente $(R P=$ 1,93). O entendimento do CFM de que o incentivo ao acompanhamento presencial do trabalho de parto, pago à parte, para o mesmo obstetra que realizou o pré-natal, funcionará como incentivo ao parto normal, está exatamente na contramão de nossos resultados, mostrando que as mulheres atendidas pelo mesmo profissional no pré-natal e parto são exatamente aquelas que apresentam as taxas de cesariana mais elevadas.

Parto; Cesárea; Remuneração; Política de Saúde 


\section{Introdução}

Ainda que a operação cesariana tenha reconhecido valor para salvaguardar a saúde da mãe e do recém-nascido, em situações em que as indicações médicas são precisas, o uso indiscriminado expõe desnecessariamente a mulher aos desfechos adversos do procedimento, com risco aumentado de morbidade 1,2,3,4 e mortalidade 5,6,7. Dados do Ministério da Saúde (Sistema de Informações sobre Nascidos Vivos. http://tabnet. datasus.gov.br/cgi/tabcgi.exe?sinasc/cnv/nvuf. def, acessado em 22/Jan/2015) mostram que os nascimentos por cesariana no Brasil aumentaram de 38,7\% em 2002 para 55,7\% em 2012.

No Brasil, as taxas de cesariana são mais altas entre as mulheres com melhores condições socioeconômicas 8,9 , de etnia branca 2 e entre aquelas que têm parto em hospitais privados 10,11,12. Nesse contexto, de acordo com Freitas et al. 2, é paradoxal que, aqueles grupos nos quais são esperadas melhores condições de saúde materna e menor risco obstétrico estejam relacionados com taxas mais elevadas de cesariana, sugerindo abuso da tecnologia médica no atendimento ao parto.

A escolha da mulher pelo parto cesáreo tem sido apontada por vários estudos como uma das razões para o aumento nas taxas de cesariana 13,14,15. Resultados de uma revisão sistemática no assunto 14 mostra uma preferência de 15,6\% por parto cesáreo, com maiores proporções em mulheres com cesariana anterior $(29,4 \%)$. A influência da mulher no processo de decisão quanto ao tipo de parto fica clara quando são examinados os resultados de um desses estudos que encontrou prevalências de cesariana mais elevadas entre as mulheres brasileiras que imigraram para Portugal, quando comparadas àquelas nativas no país em questão ${ }^{13}$. Os autores sugerem que aspectos culturais desempenham um papel importante, independentemente dos fatores médicos. Outro estudo, ao comparar fatores associados à influência do pedido da mulher na decisão do obstetra pelo parto cesáreo, em diferentes países europeus, ao encontrar extremos de 15\% na Espanha e 79\% na Inglaterra concluiu que fatores culturais e legais, relacionados com a prática obstétrica local, poderiam explicar estas diferenças 16 .

Um estudo recente 15 , conduzido com o objetivo de entender o processo de decisão pela via de parto no Brasil, mostra que entre as mulheres do setor privado $87,5 \%$ das cesáreas foram decididas no final da gestação, independentemente de complicações e indicações medicas. Ainda, de acordo com esses autores, a proporção de cesarianas foi bem maior do que o desejado pelas mulheres, tanto no setor público quanto no privado.

Em 8 de novembro de 2012, o Conselho Federal de Medicina (CFM) aprovou o Parecer CFM 39/12 17 que declara que "é ético e não configura dupla cobrança o pagamento de honorário pela gestante referente ao acompanhamento presencial do trabalho de parto". Assim, de acordo com o parecer, a disponibilidade para assistência ao parto poderá ser combinada entre as partes e cobrada pelo obstetra e, ainda, esta decisão não caracteriza lesão ao contrato estabelecido entre o profissional e a operadora do plano de saúde, que estará isenta do pagamento pelo parto.

Entende o CFM que a remuneração funciona como incentivo ao parto normal e elemento importante na reversão da elevada proporção de cesarianas no Brasil, visto que uma maior familiaridade com a gestante e sua história garantirá maior segurança ao obstetra ao decidir pelo parto normal 18. Entretanto, esse entendimento não está em sintonia com os resultados de diversos estudos mostrando que as mulheres atendidas no setor privado, aonde a probabilidade de ter o mesmo médico no pré-natal e parto é bem maior, são exatamente aquelas que apresentam as taxas de cesariana mais elevadas 19,20,21.

No estudo de Domingues et al. 15, $80 \%$ das gestantes do setor privado foram acompanhadas pelo mesmo médico durante o pré-natal e o parto. De acordo com os autores, esse fato sugere um aconselhamento favorável à cesariana, já que a decisão pela cesariana, neste grupo, não foi definida apenas por intercorrências na gestação.

Ainda que o pré-natal possa ser um momento importante para a mudança de atitudes em relação ao parto, tem sido sugerido que uma estratégia frequentemente utilizada, pelas mulheres com melhores condições socioeconômicas para obter cesariana, no setor público, é negociá-la durante o pré-natal privado 11,22 .

A participação do médico como promotor de uma cultura intervencionista já foi investigada em diversos estudos 10,14,15. Um deles observou que três em quatro das primíparas do setor privado e oito em dez do setor público, que tiveram cesarianas, gostariam de ter tido partos vaginais 15 .

Nesse contexto, forma de pagamento, por si só, não pode ser visto como determinante último das decisões pelo tipo de parto, uma vez que diferentes fatores apontam para uma maior permeabilidade do setor privado às decisões pela cesariana, por razões não estritamente médicas 14,15.

A relação entre os padrões de assistência ao parto no Brasil e as taxas de cesariana tem sido levantada por diferentes autores nas últimas décadas $2,8,9$. Gentile et al. ${ }^{9}$ postulam que não é a forma de remuneração da assistência ao parto 
isoladamente, que influencia as taxas de cesariana, mas sim uma série de fatores culturais, socioeconômicos e obstétricos, que em última análise determinam o "tipo de contrato" que se estabelece entre a paciente e o médico. No Sistema Único de Saúde (SUS), as mulheres são assistidas por diferentes profissionais ao longo do pré-natal e a assistência ao parto oferecida pelas equipes nas maternidades, trabalhando em regime de plantões. Entre as usuárias do SUS, as cesarianas são, via de regra, decididas de acordo com o diagnóstico de intercorrências durante a gestação ou trabalho de parto. Assim, no sistema público o agendamento de cesariana decorrente de acerto entre parturiente e obstetra fica bastante limitado. No sistema oferecido pelos convênios, por meio de planos de saúde, o atendimento ao pré-natal é realizado por um médico de escolha da mulher e a realização do parto por este mesmo profissional estará na dependência de acerto entre as partes, podendo ou não envolver pagamento. Por outro lado, os partos privados no Brasil, financiados pela gestante por meio de pagamento direto, é realizado por um médico de escolha da mulher, abrindo assim a possibilidade de agendamento de uma cesariana, conforme desejo da mulher e/ou indicação do obstetra.

O presente trabalho buscou investigar como a relação médico/gestante no pré-natal e a presença do mesmo médico no parto estão associadas ao tipo de parto e às decisões pelo parto cesáreo e de que maneira o incentivo à remuneração, preconizado pelo CFM, atuaria influenciando as taxas de cesariana em nosso meio.

\section{Métodos}

Um estudo com delineamento transversal selecionou uma amostra consecutiva de $600 \mathrm{mu}$ lheres tendo parto na Maternidade do Hospital Nossa Senhora da Conceição (HNSC), na cidade de Tubarão, Santa Catarina, Brasil, no período de janeiro a maio de 2014. O HNSC é um hospital centenário de caráter filantrópico, referência regional, considerado o maior hospital de Santa Catarina em número de leitos, sendo que de um total de 406 leitos, 292 estão reservados ao SUS. Foram considerados apenas os partos únicos, de nascidos vivos, visto a elevada proporção de cesarianas entre os partos gemelares.

As mulheres selecionadas com base no livro de admissão, foram contatadas no pós-parto e convidadas a participar do estudo. Apenas 6 (1\%) puérperas não puderam ser encontradas no momento da entrevista por já terem tido alta ou por estarem recebendo visitas naquele momento.
Essas foram substituídas pelos partos imediatamente posteriores.

Entrevistas estruturadas foram conduzidas com as puérperas no pós-parto imediato (24-48 horas pós-parto), no alojamento conjunto. Os questionários utilizados já haviam sido previamente validados por outros dois estudos 2,23. Este trabalho foi aprovado pelo Comitê de Ética em Pesquisa da Universidade do Sul de Santa Catarina, sob o número 24875113.9.0000.5369.

Informações a respeito das variáveis da admissão e parto (dilatação cervical na admissão, uso de indução e início do trabalho de parto) foram obtidas no livro de parto, que consiste em um registro sumarizado dos dados colhidos no momento da admissão e no pós-parto. Essa mesma fonte também foi utilizada para validar informações colhidas como parte do inquérito hospitalar: aspectos sociodemográficos, da experiência reprodutiva, da gestação e da utilização dos serviços de pré-natal incluindo idade materna, estado civil, escolaridade, partos prévios, intercorrências na gestação e frequência ao pré-natal. As demais variáveis foram coletadas como parte das entrevistas realizadas por meio de inquérito hospitalar no período pós-parto.

O enfoque maior, no presente estudo está dirigido ao entendimento de como as decisões quanto ao tipo de parto, em geral, e da cesariana, em particular, se desenvolvem do pré-natal ao parto e os fatores influenciando o desfecho representado pelo tipo de parto (cesariana ou vaginal).

A variável independente central no modelo hierárquico dos fatores influenciando tipo de parto está representada pela presença, ou não, do "mesmo médico no pré-natal e parto". As demais variáveis independentes representam aspectos demográficos e socioeconômicos: (a) idade materna (14-19 anos, 20-30 anos, 30+ anos); estado civil (casada, solteira/viúva/separada, união estável); cor da pele (branca, não branca); anos de estudos (até 8 anos completos, 9 anos ou mais); (b) experiência reprodutiva e fatores da gestação: história reprodutiva (primíparas, multípara com cesárea prévia, multípara sem cesárea prévia); gravidez planejada (sim, não); preferência por cesariana durante a gestação (sim, não); doença na gestação: definida como quaisquer condições médicas preexistentes ou iniciadas no período pré-natal que tenham exigido acompanhamento médico e/ou internação (sim, não); (c) utilização dos serviços de pré-natal: frequência ao pré-natal (0-6 consultas, 7 e mais consultas); status do pré-natal (privado, convênio, SUS); (d) admissão e parto: status do parto (privado, convênio, SUS); dilatação à admissão $(0-3 \mathrm{~cm}, 4-10 \mathrm{~cm})$; trabalho de parto (espontâneo, induzido, não). 
Inicialmente, foi investigada a distribuição das parturientes de acordo com as variáveis sociodemográficas, reprodutivas, da gestação e do parto, e a presença do mesmo médico no prénatal e parto. A seguir, razões de prevalência (RP) de cesariana, brutas e ajustadas, para todas as variáveis, foram obtidas utilizando-se análise multivariada com regressão de Cox, ajustadas pelo método de variância robusta, que mostrou fornecer estimativas corretas, sendo uma melhor alternativa na análise de estudos transversais com desfechos binários, quando comparada à regressão logística 24 . O excesso no risco $(\mathrm{RP}>1)$ foi calculado tendo como categoria de referência os grupos com menor prevalência de cesariana.

Com o objetivo de controlar para os potenciais fatores de "confundimento", cada variável foi analisada individualmente quanto ao seu efeito em relação ao desfecho (tipo de parto). Foram incluídas no modelo ajustado aquelas variáveis associadas com "tipo de parto" e "mesmo médico". As variáveis que apresentaram valor de p inferior a 0,20 foram incluídas em um modelo que buscou definir a magnitude do efeito independente da presença, no momento do parto, do "mesmo médico" que acompanhou o pré-natal, nas taxas de cesariana, quando controladas para as outras variáveis independentes.

Em nosso estudo, o cálculo do efeito ajustado se dá de acordo com um modelo teórico que busca organizar as variáveis hierarquicamente de acordo com sua posição, distal ou proximal, em relação ao desfecho 25 . Assim, as variáveis situadas nos níveis mais distais (superiores) são tratadas como confundidoras daquelas mais abaixo, enquanto aquelas situadas nos níveis mais proximais ao desfecho (níveis mais inferiores), ao mesmo tempo em que foram ajustadas para confundimento, foram também avaliadas como potenciais mediadoras das variáveis situadas mais acima no modelo. Para o modelo representando o papel mediador da presença do "mesmo médico" nas taxas de cesariana (Figura 1), as variáveis foram selecionadas como aquelas categorias de maior risco, apresentando mudanças importantes nas razões de prevalência de cesariana, ao incluir o ajuste para "mesmo médico" $\left(\mathrm{RP}_{\mathrm{AJ} 1}\right.$ para $\left.\mathrm{RP}_{\mathrm{AJ} 2}\right)$. A par do ajuste para confundimento, o foco de interesse na Figura 1 está representado pela magnitude das mudanças (efeito mediador) da variável "mesmo médico", após sua entrada no modelo hierarquizado. As análises foram conduzidas utilizando-se o programa SPSS versão 18.0 (SPSS Inc., Chicago, Estados Unidos).

\section{Cálculo do tamanho amostral}

Uma amostra consecutiva de 540 partos foi calculada como suficiente para detectar diferenças de pelo menos $50 \%(R P=1,5)$ na prevalência do desfecho (tipo do parto), de acordo com as variáveis independentes, no nível de significância estatística de $5 \%(\mathrm{p}<0,05)$. Tendo em vista eventuais perdas, um excedente de $10 \%$ foi calculado e uma amostra final de 600 puérperas foi obtida.

\section{Resultados}

Das 600 parturientes investigadas no presente trabalho, 180 (30\%) realizaram pré-natal e parto com o mesmo médico e as outras 420 (70\%) fizeram o parto com profissional diferente daquele que assistiu ao pré-natal. A taxa de cesariana entre as mulheres estudadas foi de $59,2 \%$. Entretanto, foram bem mais elevadas entre aquelas tendo o mesmo médico $(92,3 \%)$ quando comparadas às outras mulheres $(46,7 \%)$. A Tabela 1 mostra a distribuição das parturientes de acordo com o médico no pré-natal e parto e características sociodemográficas, reprodutivas, da gestação e do parto. O perfil das mulheres que tiveram o parto e pré-natal com mesmo médico, comparadas às outras parturientes, mostra uma maior proporção de gestantes com idade acima dos 30 anos, casadas, cor da pele branca, com nível mais elevado de escolaridade, com gravidez planejada e que fizeram cesariana prévia. Mais de $70 \%$ delas fizeram pré-natal e parto privados ou por convênio, comparadas àquelas que não tiveram o parto com o mesmo médico, das quais quase $80 \%$ consultaram pelo SUS. Mais de $90 \%$ das mulheres que fizeram pré-natal e parto com o mesmo médico foram admitidas com menos de $3 \mathrm{~cm}$ de dilatação e quase $80 \%$ delas não entraram em trabalho de parto. Aproximadamente $60 \%$ relataram ter preferência por cesariana no início e final da gestação, quando comparadas a 22,9\% de preferência entre as outras mulheres.

Quanto à variável "pagou pelo parto", foi detectado que esta praticamente duplicava a variável "status do parto", ou seja, as mulheres que declararam terem pago pelo parto eram, em sua quase totalidade, aquelas que tiveram o parto nos sistemas privado ou convênio, enquanto que aquelas que declararam não terem pago eram quase na mesma proporção as mesmas mulheres tendo parto pelo sistema público. Assim, a variável "pagou pelo parto" não foi incluída no modelo final, uma vez que não trazia informação adicional quanto aos efeitos da variável central 
Figura 1

Modelo hierarquizado da associação das variáveis independentes com parto cesáreo e do efeito mediador de

"mesmo médico no pré-natal e parto".

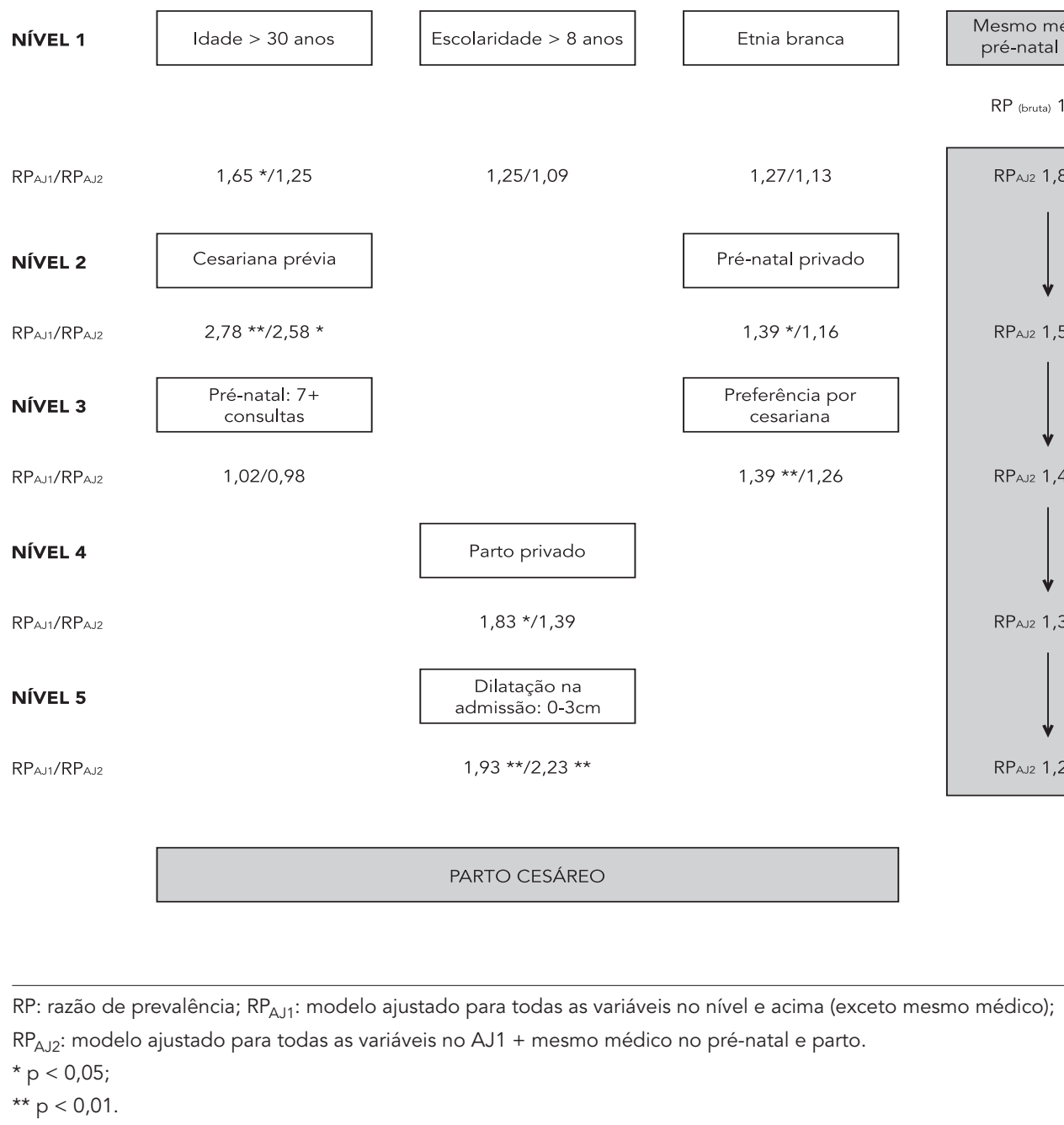

"mesmo médico" nas taxas de cesariana. Resultados, não apresentados nas tabelas, mostraram que das 51 mulheres que tiveram o parto pelo SUS, com o mesmo médico que realizou o pré-natal, apenas $6(11,7 \%)$ referiram pagar pelo parto e a totalidade destas havia feito o prénatal privado.

De acordo com a Tabela 2, as taxas de cesariana se mostraram associadas de maneira significante com "ter o mesmo médico no pré-natal e parto", com uma prevalência de parto cesáreo de quase o dobro $(\mathrm{RP}=1,98 ; \mathrm{p}<0,001)$ entre as mulheres cujo parto foi realizado pelo mesmo médico que acompanhou o pré-natal. A análise bivariada mostrou ainda razões de prevalência especialmente elevadas entre as mulheres que fi- zeram uma cesariana prévia, comparadas àquelas que tiveram apenas parto vaginal $(R P=3,04$; $\mathrm{p}<0,001)$ e entre aquelas admitidas com baixa dilatação do colo ( $R P=2,99 ; p<0,001)$. As taxas de cesariana também se mostraram aumentadas entre as mulheres que tiveram parto privado $(96 \%)$ ou por convênio $(87,8 \%)$, tendo sido praticamente o dobro nestas categorias quando comparadas às mulheres que tiveram o parto pelo SUS ( $R P=2,05$ e 1,87, respectivamente; $\mathrm{p}<0,001$ ).

As taxas foram ainda aproximadamente $70-80 \%$ maiores entre as mulheres com idade superior a 30 anos e entre aquelas que relataram preferência por cesariana no início e final da gestação $(\mathrm{RP}=1,74$ e 1,75, respectivamente; 
Distribuição das parturientes de acordo com ter o mesmo médico no pré-natal e parto e variáveis da parturiente, da gestação e do parto.

\begin{tabular}{|c|c|c|c|c|c|}
\hline \multirow[t]{3}{*}{ Variáveis } & \multicolumn{4}{|c|}{ Mesmo médico no pré-natal e parto } & \multirow[t]{3}{*}{ Valor de $\mathrm{p}$} \\
\hline & \multirow[b]{2}{*}{$\mathrm{n}$} & Sim & \multicolumn{2}{|c|}{ Não } & \\
\hline & & $\%$ & $\mathbf{n}$ & $\%$ & \\
\hline \multicolumn{6}{|l|}{ Idade materna (anos) } \\
\hline $14-19$ & 5 & 2,8 & 68 & 16,5 & $<0,001$ \\
\hline $20-30$ & 106 & 58,5 & 245 & 59,6 & \\
\hline $30+$ & 70 & 38,7 & 98 & 23,8 & \\
\hline \multicolumn{6}{|l|}{ Estado civil } \\
\hline Casada & 131 & 72,4 & 206 & 50,1 & $<0,001$ \\
\hline Solteira/Viúva/Separada & 14 & 7,7 & 37 & 9,0 & \\
\hline União estável & 36 & 19,9 & 168 & 40,9 & \\
\hline \multicolumn{6}{|l|}{ Cor da pele } \\
\hline Branca & 159 & 87,8 & 291 & 70,8 & $<0,001$ \\
\hline Não branca & 22 & 12,2 & 120 & 29,2 & \\
\hline \multicolumn{6}{|l|}{ Anos de estudos } \\
\hline Até 8 completos & 21 & 11,6 & 125 & 30,4 & $<0,001$ \\
\hline $9+$ & 160 & 80,4 & 286 & 69,6 & \\
\hline \multicolumn{6}{|l|}{ Status do pré-natal } \\
\hline Privado & 58 & 32 & 45 & 10,9 & $<0,001$ \\
\hline Convênio & 82 & 45,3 & 38 & 9,2 & \\
\hline SUS & 41 & 22,7 & 328 & 79,8 & \\
\hline \multicolumn{6}{|l|}{ Status do parto } \\
\hline Privado & 60 & 33,1 & 14 & 3,4 & $<0,001$ \\
\hline Convênio & 70 & 38,7 & 27 & 6,6 & \\
\hline SUS & 51 & 28,2 & 370 & 90 & \\
\hline \multicolumn{6}{|l|}{ Trabalho de parto } \\
\hline Espontâneo & 21 & 11,6 & 149 & 36,3 & $<0,001$ \\
\hline Induzido & 16 & 8,8 & 120 & 29,2 & \\
\hline Não & 144 & 79,6 & 142 & 34,5 & \\
\hline \multicolumn{6}{|l|}{ História reprodutiva } \\
\hline Multípara (sem cesárea prévia) & 22 & 12,1 & 130 & 31,6 & $<0,001$ \\
\hline Multípara (com cesárea prévia) & 58 & 32,1 & 92 & 22,4 & \\
\hline Primípara & 101 & 55,8 & 189 & 46 & \\
\hline \multicolumn{6}{|l|}{ Gravidez planejada } \\
\hline Sim & 118 & 65,2 & 173 & 42,1 & $<0,001$ \\
\hline Não & 63 & 34,8 & 238 & 57,9 & \\
\hline \multicolumn{6}{|l|}{ Frequência ao pré-natal (consultas) } \\
\hline $0-6$ & 13 & 7,2 & 99 & 24,1 & $<0,001$ \\
\hline $7+$ & 168 & 92,8 & 312 & 75,9 & \\
\hline \multicolumn{6}{|l|}{ Preferência por cesárea } \\
\hline Sim & 107 & 59,1 & 94 & 22,9 & $<0,001$ \\
\hline Não & 74 & 40,9 & 317 & 77,1 & \\
\hline \multicolumn{6}{|l|}{ Doença na gestação } \\
\hline Sim & 41 & 22,7 & 99 & 24,1 & 0,705 \\
\hline Não & 140 & 77,3 & 312 & 75,9 & \\
\hline \multicolumn{6}{|l|}{ Dilatação à admissão (cm) } \\
\hline $0-3$ & 147 & 90,2 & 240 & 62,5 & $<0,001$ \\
\hline $4-10$ & 16 & 9,8 & 144 & 37,5 & \\
\hline \multicolumn{6}{|l|}{ Tipo de parto } \\
\hline Cesariana & 167 & 92,3 & 188 & 45,7 & $<0,001$ \\
\hline Vaginal & 14 & 7,7 & 223 & 54,3 & \\
\hline
\end{tabular}

SUS: Sistema Único de Saúde.

Nota: dados referentes à variável "mesmo médico" não obtidos para 8 mulheres $(1,3 \%)$. 


\section{Tabela 2}

Número e taxas de cesariana, razões de prevalência brutas e intervalo de confiança para cesariana, de acordo com variáveis da parturiente, da gestação e do parto. Maternidade do Hospital Nossa Senhora da Conceição, Tubarão, Santa Catarina, Brasil.

\begin{tabular}{|c|c|c|c|c|c|}
\hline \multirow[t]{2}{*}{ Variáveis independentes } & \multicolumn{4}{|c|}{ Partos cesáreos } & \multirow[t]{2}{*}{ Valor de $p$} \\
\hline & $\mathbf{n}$ & $\%$ & RP (bruta) & IC95\% & \\
\hline \multicolumn{6}{|l|}{ Mesmo médico no pré-natal e parto } \\
\hline Sim & 167 & 92,3 & 1,98 & $1,61-2,44$ & $<0,001$ \\
\hline Não & 188 & 46,7 & 1,00 & - & - \\
\hline \multicolumn{6}{|l|}{ Idade materna (anos) } \\
\hline $14-19$ & 30 & 40,0 & 1,00 & - & 0,027 \\
\hline $20-30$ & 209 & 59,0 & 1,48 & $1,01-2,18$ & 0,043 \\
\hline $30+$ & 119 & 69,6 & 1,74 & $1,16-2,58$ & 0,008 \\
\hline \multicolumn{6}{|l|}{ Estado civil } \\
\hline Casada & 207 & 61,1 & 1,19 & $0,79-1,81$ & 0,386 \\
\hline Solteira/Viúva/Separada & 26 & 51,1 & 1,00 & - & \\
\hline União estável & 123 & 59,7 & 1,17 & $0,76-1,79$ & 0,464 \\
\hline \multicolumn{6}{|l|}{ Cor da pele } \\
\hline Branca & 290 & 63,6 & 1,35 & $1,04-1,78$ & 0,023 \\
\hline Não branca & 68 & 47,2 & 1,00 & - & - \\
\hline \multicolumn{6}{|l|}{ Anos de estudos } \\
\hline Até 8 completos & 72 & 48.3 & 1,00 & - & - \\
\hline $9+$ & 286 & 63,4 & 1,31 & $1,01-1,68$ & 0,045 \\
\hline \multicolumn{6}{|l|}{ Status do pré-natal } \\
\hline Privado & 81 & 78,6 & 1,66 & $1,27-2,16$ & $<0,001$ \\
\hline Convênio & 99 & 82,5 & 1,74 & $1,39-2,27$ & $<0,001$ \\
\hline SUS & 175 & 47,4 & 1,00 & - & $<0,001$ \\
\hline \multicolumn{6}{|l|}{ Status do parto } \\
\hline Privado & 72 & 96,0 & 2,05 & $1,52-2,63$ & $<0,001$ \\
\hline Convênio & 86 & 87,8 & 1,87 & $1,51-2,49$ & $<0,001$ \\
\hline SUS & 200 & 46,8 & 1,00 & - & - \\
\hline \multicolumn{6}{|l|}{ Trabalho de parto } \\
\hline Espontâneo & 44 & 25,6 & 1.03 & $0,671-1.656$ & 0,820 \\
\hline Induzido & 34 & 24,8 & 1,00 & - & - \\
\hline Não & 278 & 96,9 & 3.91 & $2.239-4.824$ & $<0,001$ \\
\hline \multicolumn{6}{|l|}{ História reprodutiva } \\
\hline Multípara (sem cesárea prévia) & 44 & 28,9 & 1,00 & - & - \\
\hline Multípara (com cesárea prévia) & 132 & 88,0 & 3,04 & $2,18-4,22$ & $<0,001$ \\
\hline Primípara & 182 & 61,7 & 2,13 & $1,51-2,76$ & $<0,001$ \\
\hline \multicolumn{6}{|l|}{ Gravidez planejada } \\
\hline Sim & 199 & 67,7 & 1,27 & $1,03-1,56$ & 0,025 \\
\hline Não & 159 & 52,8 & 1,00 & - & - \\
\hline \multicolumn{6}{|l|}{ Frequência ao pré-natal (consultas) } \\
\hline $0-6$ & 55 & 45,8 & 1,00 & - & - \\
\hline $7+$ & 303 & 63,1 & 1,37 & $0,99-1,755$ & 0,053 \\
\hline \multicolumn{6}{|l|}{ Preferência por cesariana } \\
\hline Sim & 170 & 83,3 & 1,75 & $1,44-2,18$ & $<0,001$ \\
\hline Não & 188 & 47,5 & 1,00 & - & - \\
\hline \multicolumn{6}{|l|}{ Pediu para ter cesariana no pré-natal } \\
\hline Sim & 111 & 99,1 & 1,22 & $0,86-1,65$ & 0,297 \\
\hline Não & 55 & 80,9 & 1,00 & - & - \\
\hline \multicolumn{6}{|l|}{ Dilatação à admissão (cm) } \\
\hline $0-3$ & 286 & 73,3 & 2,99 & $2,15-4,16$ & $<0,001$ \\
\hline $4-10$ & 38 & 23,0 & 1,00 & - & - \\
\hline
\end{tabular}

IC95\%: intervalo de 95\% de confiança; RP: razão de prevalência; SUS: Sistema Único de Saúde.

Nota: dados referentes à variável "mesmo médico" não obtidos para 8 mulheres (1,3\%). 
$\mathrm{p}<0,05)$. Escolaridade mais elevada e cor da pele branca, também se mostraram associadas à maior ocorrência de cesariana, ainda que com menor intensidade. Por outro lado, maior frequência ao pré-natal e pedido de cesariana durante gestação, ainda que tenham apresentado algum efeito, não alcançaram significância estatística na análise bivariada.

A Tabela 3 apresenta as razões de prevalência para taxas de cesariana ajustadas por todas as variáveis no modelo exceto "mesmo médico no pré-natal e parto" (AJ1) e por todas as variáveis no modelo + "mesmo médico no pré-natal e parto" (AJ2). Após o primeiro ajuste que incluiu todas as variáveis no modelo, exceto "mesmo médico no pré-natal e parto", as categorias de idade acima dos 30 anos, pré-natal e parto realizados no sistema privado ou por convênio, cesariana prévia, primiparidade, preferência por cesariana e baixa dilatação à admissão, mantiveram efeitos importantes e estatisticamente significantes ( $R P>1,5 ; \mathrm{p}<0,05)$. Por outro lado, cor da pele branca, grau mais elevado de escolaridade, gravidez planejada, maior frequência ao pré-natal e solicitar cesariana, perderam força e significância estatística.

A variável "trabalho de parto", ainda que não tenha mostrado diferença significante nas taxas de cesariana, ao comparar as mulheres com parto espontâneo e induzido ( $\mathrm{RP}=1,06 ; \mathrm{p}=0,83$ ) apresentou uma ocorrência de cesariana de mais do que o triplo entre as mulheres que não entraram em trabalho de parto, quando comparadas àquelas que tiveram parto espontâneo. A força e significância dessa associação não foi afetada ao ajustar pela variável "mesmo médico" $(\mathrm{RP}=$ $3,28 ; \mathrm{p}<0,01)$.

Ainda, de acordo com os resultados na Tabela 3, após incluir no modelo a variável "mesmo médico no pré-natal e parto”, apenas as variáveis: cesariana prévia, primiparidade e baixa dilatação à admissão, mantiveram um efeito independente nas taxas de cesariana, tendo conservado a força da associação e alta significância estatística comparadas ao modelo anterior $(\mathrm{RP}>1,8 ; \mathrm{p}<0,001)$. Ter o mesmo médico no pré-natal e parto, variável central no modelo final, conservou associação independente e estatisticamente significante com as taxas de cesariana, após ajuste para todas as variáveis no modelo $(\mathrm{RP}=1,98$; IC: 1,61-2,44), mostrando ter sido parcialmente mediada pelo efeito das outras variáveis (confundidoras), mediação esta expressa na queda da RP(bruta) para $\mathrm{RP}_{\mathrm{AJ} 2}(\mathrm{de} \mathrm{RP}=1,98$ para $\mathrm{RP}=1,33 ; \mathrm{p}<0,05)$.

A magnitude do efeito mediador da variável "mesmo médico no pré-natal e parto", sobre as outras variáveis no modelo, está representada pela magnitude das mudanças nas razões de prevalência, após a entrada da variável "mesmo médico" no modelo, conforme apresentado na Tabela 3. As maiores quedas, na associação com as taxas de cesariana, após levar em conta o efeito de "mesmo médico", foram registradas para as variáveis: parto privado (RP de 1,83 para 1,39 ), parto por convênio (RP de 1,73 para 1,28), pré-natal privado (RP de 1,39 para 1,16), pré-natal por convênio (RP de 1,43 para 1,16) e idade acima dos 30 anos (RP de 1,65 para 1,25).

A Figura 1 apresenta os resultados para o efeito, de cada uma das variáveis, nas taxas de cesariana, de acordo com o ajuste por níveis hierárquicos de influência. As variações nas RPs são apresentadas, tanto ao comparar, no sentido horizontal, os dois modelos de ajuste (sem e com a influência de mesmo medico no pré-natal e parto) quanto para mudanças no efeito da presença no parto do mesmo médico que realizou o prénatal, ajustado por nível hierárquico de influência (sentido vertical), resultados já descritos na Tabela 3. Os resultados apresentados na Figura 1 mostram que a variável "mesmo médico" manteve o efeito independente nas taxas de cesariana e a significância estatística, ao longo do modelo hierárquico.

Diferenças nas razões de prevalência a partir do nível mais distal (RP bruta) até o desfecho (parto cesáreo) apontam para mediações importantes do nível de influência das variáveis sociodemográficas $(\mathrm{RP}=1,98$ para $\mathrm{RP}=1,84)$, reprodutivas $(R P=1,84$ para $R P=1,52)$, do pré-natal e das preferências ( $R P=1,52$ para $R P=1,43)$, da categoria social do parto $(\mathrm{RP}=1,43$ para $\mathrm{RP}=1,39)$ e finalmente do status do parto no momento da admissão ( $\mathrm{RP}=1,39$ para $\mathrm{RP}=1,28)$.

\section{Discussão}

A questão das altas taxas de cesariana tem sido objeto de crescente discussão, visto sua elevação contínua, tanto no Brasil quanto internacionalmente 19,21. Nesse quadro, o Brasil tem sido citado frequentemente como líder na utilização da cesariana, sobre a qual fatores socioculturais, institucionais, financeiros e da prática obstétrica estariam por trás da assim denominada "cultura da cesariana no Brasil" 8.

A taxa de cesariana entre as 600 mulheres estudadas foi de $59,2 \%$, resultado muito próximo dos 55,7\% reportados para todo país em 20125 . Taxas de cesariana mais elevadas no setor privado comparado ao setor público têm sido amplamente divulgadas por diferentes estudos 2,19,20,21. Nossos resultados mostraram prevalências de cesariana de quase o dobro entre as mulheres que tiveram parto privado ou por convênio 
Tabela 3

Taxas de cesariana, razões de prevalência ajustadas e intervalo de confiança para cesariana, de acordo com variáveis da parturiente, da gestação e do parto. Maternidade do Hospital Nossa Senhora da Conceição, Tubarão, Santa Catarina, Brasil.

\begin{tabular}{|c|c|c|c|c|c|c|c|}
\hline Variáveis & Cesarianas (\%) & $\mathrm{RP}_{\mathrm{AJ} 1}$ & IC95\% & Valor de $p$ & $\mathrm{RP}_{\mathrm{AJ} 2}$ & IC95\% & Valor de $p$ \\
\hline \multicolumn{8}{|l|}{ Mesmo médico no pré-natal e parto } \\
\hline Sim & 92,3 & 1,98 & $1.61-2,44$ & $<0,001$ & 1,33 & $1,07-1,69$ & 0,044 \\
\hline Não & 45,7 & 1,00 & - & - & 1,00 & - & - \\
\hline \multicolumn{8}{|l|}{ Idade materna (anos) } \\
\hline $14-19$ & 40,0 & 1,00 & - & - & 1,00 & - & - \\
\hline $20-30$ & 59,0 & 1,40 & $0,95-2,06$ & 0,086 & 1,18 & $0,80-1,75$ & 0,404 \\
\hline $30+$ & 69,6 & 1,65 & $1,09-2,49$ & 0,013 & 1,25 & $0,83-1,89$ & 0,236 \\
\hline \multicolumn{8}{|l|}{ Cor da pele } \\
\hline Branca & 63,6 & 1,27 & $0,98-1,67$ & 0,075 & 1,13 & $0,86-1,49$ & 0,362 \\
\hline Não branca & 47,2 & 1,00 & - & - & 1,00 & - & - \\
\hline \multicolumn{8}{|l|}{ Anos de estudos } \\
\hline Até 8 completos & 48,3 & 1,00 & - & - & 1,00 & - & - \\
\hline $9+$ & 63,4 & 1.25 & $0,96-1,62$ & 0,09 & 1.09 & $0,83-1,43$ & 0,386 \\
\hline \multicolumn{8}{|l|}{ Status do pré-natal } \\
\hline Privado & 78,6 & 1,39 & $1,05-1,85$ & 0,019 & 1,16 & $0,86-1,58$ & 0,317 \\
\hline Convênio & 82,5 & 1,43 & $1,09-1,88$ & 0,006 & 1,16 & $0,87-1,61$ & 0,278 \\
\hline SUS & 47,2 & 1,00 & - & 0,012 & 1,00 & - & 0,503 \\
\hline \multicolumn{8}{|l|}{ Status do parto } \\
\hline Privado & 96,0 & 1,83 & $1,34-2,46$ & $<0,001$ & 1,39 & $0,63-2,99$ & 0,376 \\
\hline Convênio & 87,8 & 1,73 & $1,22-2,41$ & 0,002 & 1,28 & $0,48-2,76$ & 0,498 \\
\hline SUS & 46,8 & 1,00 & - & - & 1,00 & & \\
\hline \multicolumn{8}{|l|}{ Trabalho de parto } \\
\hline Sim & 25,6 & 1.06 & $0,67-1.66$ & 0,82 & 1,05 & $0,69-1.68$ & 0,83 \\
\hline Induzido & 24,8 & 1,00 & - & - & 1,00 & - & - \\
\hline Não & 96,9 & 3.28 & $2.24-4.82$ & $<0,001$ & 3.23 & $2.07-4.74$ & $<0,001$ \\
\hline \multicolumn{8}{|l|}{ História reprodutiva } \\
\hline Multípara (sem cesárea prévia) & 28,9 & 1,00 & - & - & 1,00 & - & - \\
\hline Multípara (com cesárea prévia) & 88,0 & 2,78 & $2,01-3,68$ & $<0,001$ & 2,58 & $1,79-3,48$ & $<0,001$ \\
\hline Primípara & 61,7 & 2,01 & $1,29-2,63$ & $<0,001$ & 1,83 & $1,29-2,39$ & 0,001 \\
\hline \multicolumn{8}{|l|}{ Gravidez planejada } \\
\hline Sim & 67,7 & 1,09 & $0,87-1,37$ & 0,438 & 1,06 & $0,847-1,34$ & 0,612 \\
\hline Não & 52,8 & 1,00 & - & - & 1,00 & - & - \\
\hline \multicolumn{8}{|l|}{ Frequência ao pré-natal (consultas) } \\
\hline $0-6$ & 45,8 & 1,00 & - & - & 1,00 & - & - \\
\hline $7+$ & 63,1 & 1,02 & $0,76-1,38$ & 0,885 & 0,98 & $0,72-1,33$ & 0,899 \\
\hline \multicolumn{8}{|l|}{ Preferência por cesariana } \\
\hline Sim & 83,3 & 1,39 & $1,11-1,76$ & 0,05 & 1,26 & $0,99-1,60$ & 0,061 \\
\hline Não & 47,5 & 1,00 & - & - & 1,00 & - & - \\
\hline \multicolumn{8}{|l|}{ Dilatação à admissão $(\mathrm{cm})$} \\
\hline $0-3$ & 73,3 & 1,93 & $1,28-2,89$ & 0,005 & 2,23 & $1,58-3,11$ & $<0,001$ \\
\hline $4-10$ & 23,1 & 1,00 & - & - & 1,00 & - & - \\
\hline
\end{tabular}

IC95\%: intervalo de 95\% de confiança; $\mathrm{RP}_{\mathrm{AJ} 1}$ : ajuste para todas as variáveis no nível hierárquico e acima, exceto mesmo "médico no pré-natal e parto"; $\mathrm{RP}_{\mathrm{AJ} 2}$ : ajuste $\mathrm{RP}_{\mathrm{AJ} 1}+$ mesmo médico no pré-natal e parto; SUS: Sistema Único de Saúde. 
quando comparadas àquelas atendidas pelo SUS (91,3\% vs. 46,8\%).

Resultados mostrando uma maior ocorrência de cesarianas entre as mulheres acima dos 30 anos, naquelas com história de cesariana prévia, com maior frequência ao pré-natal e entre aquelas admitidas com baixa dilatação do colo, também têm sido citados por outros autores 11,26,27,28.

Ao dirigir nossa atenção para as variáveis sociais, as taxas de cesariana foram mais altas entre as mulheres de cor da pele branca, de escolaridade mais elevada e entre aquelas que fizeram consultas pré-natal e partos no setor privado ou por intermédio de convênio, ou seja, entre aquelas potencialmente com melhores condições socioeconômicas, dentre as quais se espera um menor risco obstétrico. Resultados de outros estudos apontam na mesma direção e reforçam a suspeita de que decisões, não estritamente médicas, entre as mulheres com melhores condições sociais explicam essas diferenças 22,27.

Outras variáveis cuja influência na ocorrência de cesariana foi explorada, por outros estudos, ainda que em menor grau, são gravidez planejada e preferência por tipo de parto, e se mostraram associadas a uma maior ocorrência de parto cesáreo neste estudo.

Após o ajuste para todas as variáveis no modelo final, cesariana prévia ( $\mathrm{RP}=2,58$ ), admissão precoce ao parto $(R P=2,23)$ e presença no parto do mesmo médico que realizou o pré-natal $(R P=1,28)$ mantiveram efeito e significância estatística.

Após o ajuste final não foi encontrada diferença significante nas taxas de cesariana, ao comparar as mulheres com parto espontâneo e induzido. Por outro lado, taxas de cesariana de mais do que o triplo entre as mulheres que não entraram em trabalho de parto permaneceram inalteradas após ajuste pela variável "mesmo médico". Esses resultados, somados ao fato de que $80 \%$ das mulheres assistidas pelo mesmo médico no pré-natal e parto não entraram em trabalho de parto (Tabela 1), sugerem que, muito provavelmente, para a grande maioria destas mulheres a decisão pela cesariana já havia ocorrido antes mesmo da admissão.

Em nosso estudo, a variável "mesmo médico no pré-natal e parto" ocupa lugar central no modelo teórico das influências circundando as decisões pelo tipo de parto, representado na $\mathrm{Fi}$ gura 1. No modelo teórico conceitual, essa variável representa a manutenção da relação médico/gestante, desde o pré-natal até o momento do parto, e tem como objetivo maior investigar a influência desta relação nas decisões quanto ao tipo de parto. A manutenção de um efeito in- dependente sugere uma maior permeabilidade do setor privado às decisões pela cesariana, por razões não estritamente médicas, já destacada em outros trabalhos 22,26.

Resultados deste e de outros estudos $2,8,9,10,11,22$ apontam para as desigualdades sociais na gestação e parto, e ressaltam o papel da relação médico/paciente nas chances de decisão por uma cesariana não suportada por indicações estritamente médicas.

Embora uma razão frequentemente apontada pela classe obstétrica para justificar um aumento nas taxas de cesariana seja a preferência e o pedido das mulheres $13,14,15$, tem sido mostrado que a prevalência de cesarianas é muito maior do que a proporção de mulheres que desejam este tipo de parto 15 . Nossos resultados corroboram essas afirmações. Em nosso estudo, a taxa de cesarianas entre as mulheres que declararam preferência pelo parto vaginal foi de $72,1 \%$ no sistema privado/convênio e 33,6\% no sistema publico. Ainda, aproximadamente $60 \%$ das mulheres que fizeram o parto com o mesmo médico que realizou o pré-natal relataram preferência por cesariana no início e final da gestação, comparadas a $22,9 \%$ de preferência entre as outras mulheres. Entretanto, o efeito de preferir cesariana na ocorrência de parto cesáreo perdeu significância após ajuste no modelo final, mostrando ser mediada por categoria (pública ou privada) do parto e mesmo médico no pré-natal e parto.

Um estudo conduzido em Pelotas, no sul do Brasil 22, conclui que uma taxa de cesarianas mais elevada entre as mães de melhor nível de escolaridade, mesmo no setor público, poderia ser explicada tanto por uma maior capacidade de persuasão por parte destas mulheres quanto pelo fato dos obstetras perceberem estas pacientes como "privadas" e consequentemente merecedoras de uma cesariana.

A organização da assistência obstétrica no Brasil por parte do SUS em que as mulheres são, via de regra, acompanhadas por diferentes profissionais no pré-natal e parto, em sistema de plantão, a princípio limita a possibilidade da escolha da mulher sobre o tipo de parto ${ }^{15}$. Assim, diferenças encontradas nas taxas de cesariana em favor das mulheres com melhor nível social (representado por escolaridade mais elevada) mesmo entre as pacientes do SUS, conforme referido por Barros et al. ${ }^{22}$ podem ser imputadas a outros fatores não necessariamente ligados às indicações estritamente médicas.

Em nosso estudo, ainda que as mulheres pertencentes ao nível mais elevado de escolaridade tenham apresentado taxas mais elevadas de cesariana, este efeito se mostrou totalmente mediado por categoria (pública ou privada) do 
parto, desaparecendo após ajuste por esta variável. Assim, diferentes fatores, relacionados à organização obstétrica no setor privado, apontam para decisões quanto ao tipo de parto não necessariamente baseadas em indicações clínicas $23,26,27,28$, entre estes a frequentemente aludida conveniência para a agenda do obstetra 11.

Neste estudo, as mulheres que fizeram o parto com o mesmo médico que acompanhou o pré-natal pertenciam aos grupos de idade mais avançada, de maior escolaridade, com maior frequência ao pré-natal, fazendo pré-natal e parto privado ou por convênio, e admitidas mais precocemente, ou seja, exatamente os grupos com as taxas mais elevadas de cesariana. A prevalência por parto cesáreo entre essas mulheres foi mais do que o dobro (92,3\%) se comparadas àquelas que fizeram o parto com um médico que não aquele que acompanhou o pré-natal $(45,7 \%)$.

De acordo com Brennan et al. 29 , atualmente nenhum sistema em particular que busque definir taxas ideais para cesarianas medicamente indicadas pode ser aplicado universalmente, principalmente devido às variações nas características das populações estudadas, diferenças inter e intrainstitucionais na prática obstétrica e nas definições das indicações absolutas para cesariana ${ }^{29}$. Diferentes razões para o aumento progressivo e quase universal nas taxas de cesariana nas últimas décadas têm sido propostas, incluindo maior aceitação pelo obstetra da cesariana a pedido 11,15 , aumento nas preocupações médico-legais 30, incremento no uso de cesariana entre as mulheres obesas sem outras morbidades 31 , aumento da idade média das gestantes 32 e escolha pela cesariana eletiva ${ }^{33}$. Nesse contexto, ainda que o limite de $15 \%$ preconizado pela Organização Mundial da Saúde (OMS) nos anos 197034 siga sendo amplamente utilizado por muitos autores nos dias de hoje, como limite aceito para cesarianas com indicações médicas, mudanças temporais na "cultura do parto" juntamente com o conhecimento acumulado nas últimas décadas sugerem que este parâmetro deva ser reconsiderado. Por outro lado, no presente estudo, diferenças expressivas nas taxas de cesariana ao comparar os sistemas público e privado e entre mulheres tendo ou não o mesmo médico no pré-natal e parto, por si só, apontam para um excesso no uso da cesariana em nosso meio.

A análise de acordo com o modelo hierárquico traz informação essencial a respeito do efeito mediador de "mesmo médico" no momento do parto, ao conduzir o ajuste por esta variável. Ter o mesmo médico no pré-natal e parto esteve associado com a ocorrência de parto cesáreo; resultados mostram manutenção de parte do efeito após ajuste por todas as variáveis classicamente associadas às taxas de cesariana. Por outro lado, variações na influência (RP) das diferentes variáveis, ao ajustar por "mesmo médico" ao longo do modelo, apontam para o potencial efeito de indicações relativas ou não estritamente médicas na decisão final pelo tipo de parto. Dessa forma, ainda que os resultados mostrem manutenção de parte do efeito, após ajuste por todas as variáveis classicamente associadas às taxas de cesariana, uma queda importante, após o ajuste, mostra que grande parte do efeito da variável "mesmo médico" pode ser explicada por outras variáveis influenciando a decisão pelo tipo de parto. Nesse sentido, modificações importantes no risco ao ajustar para cesariana prévia, maior frequência ao pré-natal, preferência da mulher por cesariana e admissão precoce ao parto, trazem à tona os efeitos da preferência da mulher influenciando as decisões do obstetra e seu reflexo em uma maior ocorrência de cesarianas decididas eletivamente como já destacado em outros estudos 13,14,15. Ainda, a manutenção do efeito independente de cesariana prévia e de admissão precoce ao parto, após ajuste para todas as variáveis no modelo, aponta por um lado para a já reportada conveniência para o obstetra ao decidir-se precocemente por um parto que poderia "arrastarse", e por outro, reforça o postulado por Craigin em 1916 35: "uma vez cesárea, sempre cesárea".

Algumas variáveis, potencialmente associadas com uma maior prevalência de cesariana não foram consideradas no presente trabalho. Informação sobre "posição fetal” disponível apenas nos prontuários médicos não foi registrada para nenhuma das mulheres com "ausência de trabalho de parto" e raramente constava nos prontuários para as outras mulheres, assim não pôde ser incluída no modelo. A variável "idade gestacional” não foi incluída, uma vez que uma relação entre o uso crescente da cesariana e um aumento dos nascimentos prematuros tem sido largamente discutida, sendo sugerido que um incremento na prevalência de bebês prematuros possa estar relacionado a um aumento nas cesarianas decididas eletivamente. Em nosso estudo, aproximadamente $70 \%$ dos prematuros, tendo o parto com o "mesmo médico", não entraram em trabalho de parto sugerindo potencial para "causalidade reversa”. Consequentemente, a variável "peso ao nascer", pela sua estreita relação com idade gestacional, também não foi incluída. Entretanto, a larga diferença na taxa de cesarianas, ao comparar mulheres tendo ou não o "mesmo médico", sugere que estas três variáveis isoladamente não mudariam de maneira importante o quadro observado.

Neste estudo, conclusões a respeito de pagamento à parte, entre as mulheres com plano 
de saúde, ficaram prejudicadas uma vez que não foi possível separar "pagamento à parte" daqueles efetuados como parte do convênio. Entre as mulheres do sistema privado, por sua vez, já está acordado desde o início do pré-natal que aquele que acompanha o pré-natal será o obstetra "pago" pelo parto. Assim, ainda que a variável "pagamento pelo parto" não tenha permitido conclusões maiores referentes às mulheres dos sistemas privado/convênio, uma vez que praticamente duplicava a variável "status do parto", resultados mostrando que quase $90 \%$ das mulheres tendo parto pelo SUS com o mesmo médico que realizou o pré-natal declararam não ter pago pelo parto e que, a totalidade daquelas que referiram pagar haviam realizado pré-natal privado, deixam claro que o "pagamento à parte" pelo parto não é condição sine-qua-non para ter o mesmo médico e apontam para o "potencial para negociação" paciente/obstetra, já referido por outros estudos.
Ainda que possa ser argumentado que a presença do mesmo médico no pré-natal e parto esteja potencialmente associada com uma menor incidência de complicações no pós-parto 2 , não seria lógico ou ético utilizá-lo para justificar a decisão por um procedimento cirúrgico, sem indicações médicas estritas, quando a viabilidade para o parto normal puder ser antecipada.

$\mathrm{O}$ entendimento do CFM, por meio do $\mathrm{Pa}$ recer CFM 39/12 17, de que o acompanhamento presencial do trabalho de parto pelo mesmo médico que realizou o pré-natal funcionará como incentivo ao parto normal, está exatamente na contramão dos resultados aqui apresentados, assim como os de diversos outros autores mostrando que as mulheres atendidas pelo mesmo médico no pré-natal e parto são exatamente aquelas que apresentam as taxas de cesariana mais elevadas 2,15,19,22. 


\section{Resumen}

El estudio investiga cómo el pago por parto, recomendado por el Consejo Federal de Medicina de Brasil (CFM), influye en las tasas de cesárea. Se investigó una muestra consecutiva de 600 mujeres embarazadas. La tasa de cesárea en la muestra fue de un 59,2\% y un 92,3\% entre las mujeres con parto y atención prenatal con el mismo profesional. Las tasas de cesáreas fueron significativamente mayores en los grupos exactamente con mayor prevalencia del mismo profesional, es decir, edad avanzada $(R P=1,65)$, educación superior $(R P=1,25)$, atención prenatal privada $(R P=1,39) o$ por acuerdo $(R P=1,43)$, cesárea anterior $(R P=2,78)$ y admisión precoz $(R P=1,93)$. La recomendación del CFM que incentiva la atención del parto por el mismo profesional que realizó la atención prenatal, es exactamente la contraria a la nuestra, debido a nuestros resultados, ya que demuestra que las mujeres atendidas por el mismo profesional en la atención prenatal y el parto son exactamente las que presentan las tasas más altas de cesárea.

Parto; Cesárea; Remuneración; Política de Salud

\section{Colaboradores}

P. F. Freitas foi responsável pela concepção e redação do artigo, participou de todas as etapas de produção e da versão final a ser publicada. B. C. Moreira participou da análise dos dados, da redação do artigo e da versão final a ser publicada. A. L. Manoel participou da análise, interpretação dos dados e aprovação da versão final a ser publicada. A. C. A. Botura participou da redação e aprovação da versão final a ser publicada.

\section{Agradecimentos}

Ao Programa de Incentivo à Pesquisa PUIC/UNISUL.

\section{Referências}

1. Liu S, Liston RM, Joseph KS, Heaman M, Sauve R, Kramer MS. Maternal mortality and severe morbidity associated with low risk planned cesarean delivery versus planned vaginal delivery at term. CMAJ 2007; 13:455-60.

2. Freitas PF, Savi EP. Desigualdades sociais nas complicações da cesariana: uma análise hierarquizada. Cad Saúde Pública 2011; 27:2009-20.

3. Van Dillen J, Zwart JJ, Schuttle J, Bloemenkamp KW, van Roosmalen J. Severe acute morbidity and mode of delivery in the Netherlands. Acta Obstet Gynecol Scand 2010; 89:1460-5.
4. Acosta CD, Kurinczuk JJ, Lucas DN, Tuffnell D, Sellers S, Knight M. Severe maternal sepsis in the UK, 2011-2012: a national case-control study. PLoS Med 2014; 11:1001672.

5. Harper MA, Byington RP, Espeland MA, Naughyon M, Meyer R, Lane K. Pregnancy-related death and health care services. Obstet Gynecol 2003; 102:2738.

6. Deneux-Tharaux C, Carmona E, Bouvier-Colle M, Béart F. Postpartum maternal mortality and cesarean delivery. Obstet Gynecol 2006; 108 (3 Pt 1):541-8. 
7. Kilsztajn S, Carmo MS, Machado LC, Lopes ES, Lima LZ. Caesarean sections and maternal mortality in São Paulo. Eur J Obstet Gynecol Reprod Biol 2007; 132:64-9.

8. Freitas PF, Drachler M, Leite J, Grassi P. Social inequalities in caesarean section rates in primiparous. J Public Health 2005; 39:761-7.

9. Gentile FP, Noronha Filho G, Cunha AA. Associação entre a remuneração da assistência ao parto e a prevalência de cesariana em maternidades do Rio de Janeiro: uma revisão da hipótese de Carlos Gentile de Mello. Cad Saúde Pública 1997; 13:221-6.

10. Puccini R, Pedroso GC, Silva EMK, Araújo NS, Silva NN. Eqüidade na atenção pré-natal e ao parto em área da Região Metropolitana de São Paulo, 1996. Cad Saúde Pública 2003; 19:35-45.

11. Behague DP, Victora CG, Barros FC. Consumer demand for caesarean sections in Brazil: informed decision making, patient choice, or social inequality? A population based birth cohort study linking ethnographic and epidemiological methods. BMJ 2002; 324:942-5.

12. Victora C, Vaughan J, Barros F, Silva A, Tomasi E. Explaining trends in inequities: evidence from Brazilian child health studies. Lancet 2000; 356:1093-8.

13. Teixeira C, Correia S, Victora CG, Barros H. The Brazilian preference: cesarean delivery among immigrants in Portugal. PLoS One 2013; 8:e60168.

14. Mazzoni A, Althabe F, Liu NH, Bonotti AM, Gibbons L, Sánchez AJ, et al. Women's preference for caesarean section: a systematic review and meta-analysis of observational studies. BJOG 2011; 118:391-9.

15. Domingues RMSM, Dias MAB, Nakamura-Pereira M, Torres JA, d'Orsi E, Pereira APE, et al. Processo de decisão pelo tipo de parto no Brasil: da preferência inicial das mulheres à via de parto final. Cad Saúde Pública 2014; 30 Suppl 1:S101-16.

16. Habiba M, Kaminski M, Da Fre M, Marsal K, Bleker O, Librero J, et al. Caesarean section on request: a comparison of obstetricians' attitudes in eight European countries. BJOG 2006; 113:647-56.

17. Conselho Federal de Medicina. Parecer CFM 39/12. http://www.portalmedico.org.br/parece res/CFM/2012/39_2012.pdf (acessado em 23/Dez/ 2014).

18. Conselho Federal de Medicina. Parecer considera ética a remuneração. Medicina Publicação Oficial do Conselho Federal de Medicina 2012; ano XXVII, no 214. http://www.cadastro.abneuro.org/site/jor nal214CFM.pdf.

19. Patah LEM, Malik AM. Modelos de assistência ao parto e taxa de cesárea em diferentes países. Rev Saúde Pública 2011; 45:185-94.

20. Souza Jr. JC, Kunkel N, Gomes MA, Freitas PF. Equidade inversa e desigualdade no acesso a tecnologia no parto em Santa Catarina. Rev Bras Saúde Matern Infant 2007; 7:387-403.
21. Belizan JM, Althabe F, Barros FC, Alexander S. Rates and implications of caesarean sections in Latin America: ecological study. BMJ 1999; 319:1397402.

22. Barros AJ, Santos IS, Matijasevich A, Domingues MR, Silveira M, Barros FC, et al. Patterns of deliveries in a Brazilian birth cohort: almost universal cesarean sections for the better-off. Rev Saúde Pública 2011; 45:635-43.

23. Freitas PF. The epidemic of caesarean sections in Brazil, factors influencing type of delivery in Florianópolis, South Brazil [Doctoral Dissertation]. London: London School of Hygiene and Tropical Medicine; 2000.

24. Barros AJ, Hirakata VN. Alternatives for logistic regression in cross-sectional studies: an empirical comparison of models that directly estimate the prevalence ratio. BMC Med Res Methodol 2003; 3:21.

25. Camacho LAB. Curso de epidemiologia clínica: viés e confundimento. http://www.fiocruz.br/pes quisaclinica/media/IPEC-ViesConfund.pdf (acessado em 17/Jun/2014).

26. Hopkins K. Are Brazilian women really choosing to delivery by cesarean? Soc Sci Med 2000; 51:725-40.

27. Sakae TM, Freitas PF, d'Orsi E. Fatores associados a taxas de cesárea em hospital universitário. Rev Saúde Pública 2009; 43:472-80.

28. Freitas PF, Drachler ML, Leite JC, Marshall T. Inequalities in cesarean delivery rates by ethnicity and hospital accessibility in Brazil. Int J Gynaecol Obstet 2009; 107:198-201.

29. Brennan DJ, Robson MS, Murphy M, O’Herlihy C. Comparative analysis of international cesarean delivery rates using 10-group classification identifies significant variation in spontaneous labor. Am J Obstet Gynecol 2009; 201:308.e1-8.

30. Minkoff H, Chervenak FA. Elective primary cesarean delivery. N Engl J Med 2003; 348:946-50.

31. Poobalan AS, Aucott LS, Gurung T, Smith WC, Bhattacharya S. Obesity as an independent risk factor for elective and emergency caesarean delivery in nulliparous women: systematic review and meta-analysis of cohort studies. Obes Rev 2009; 10:28-35.

32. Lin HC, Sheen TC, Tang CH, Kao S. Association between maternal age and the likelihood of a cesarean section: a population-based multivariate logistic regression analysis. Acta Obstet Gynecol Scand 2004; 83:1178-83.

33. Zhang J, Landy HJ, Branch DW, Burkman R, Haberman S, Gregory KD, et al. Contemporary patterns of spontaneous labor with normal neonatal outcomes. Obstet Gynecol 2010; 116:1281-7.

34. Appropriate technology for birth. Lancet 1985; 2:436-7.

35. Craigin E. Conservatism in obstetrics. N Y State J Med 1916; 104:1-3.

Recebido em 13/Jan/2015

Versão final reapresentada em 31/Mar/2015

Aprovado em 20/Abr/2015 


\section{Comentário sobre o artigo de Freitas et al.}

Comment on the paper by Freitas

et al.

Comentario acerca del artículo de

Freitas et al.

http://dx.doi.org/10.1590/0102-311XC0010915

Maria do Carmo Leal

Escola Nacional de Saúde Pública Sergio Arouca, Fundação Oswaldo Cruz Rio de Janeiro, Brasil. ducaleal@gmail.com
Muito importante einteressante, no contexto atual do país, o artigo de Freitas et al. que se intitula O Parecer do Conselho Federal de Medicina, o Incentivo à Remuneração ao Parto e as Taxas de Cesariana no Brasil, cujo título me surpreendeu por não imaginar como poderiam ter realizado um estudo sobre este parecer e suas consequências sobre as taxas de cesariana no Brasil, dado que o referido parecer não foi referendado pela Agência Nacional de Saúde Suplementar (ANS), conforme nota no 394/2014/GEAS/GGRAS/DIPRO/ ANS, de 15 de maio de 2014.

Na verdade, os autores não examinaram exatamente essas relações nesse artigo, mas utilizaram a temática do parecer do Conselho Federal de Medicina (CFM) para notificar a sua insuficiência ou inadequação para reverter ou mesmo diminuir as taxas de cesarianas, dado que o maior risco para a ocorrência de uma cesariana é o atendimento ao parto ser realizado pelo mesmo médico que acompanhou a gestante durante o pré-natal. Nesse sentido, o parecer do CFM não contribuiria para reduzir o problema porque reforça o modelo atual de atenção ao parto que tem mantido um crescimento anual de dois pontos percentuais na taxa de cesariana.

Achados muito semelhantes ao desse estudo de Freitas et al. já haviam também sido encontrados por Torres 1 com dados da pesquisa Nascer no Brasil. A referida autora, em sua tese de doutorado, comparou os resultados alcançados por um hospital privado da Região Sudeste, que tinha um modelo atípico de atenção ao parto, com a amostra de hospitais privados da pesquisa na mesma região, que seguia o modelo atual de agendamento prévio das cesarianas, denominado de modelo de conveniência obstétrica. O hospital atípico caracterizava-se por oferecer uma atenção obstétrica centrada no trabalho de equipes formadas por médicos e enfermeiros obstétricos, tanto durante o pré-natal quanto no momento do parto, além de um diálogo permanente com as gestantes sobre as vantagens de um parto vaginal. Houve, portanto, uma quebra nessa relação de exclusividade da gestante com o seu pré-natalista, bem como foi introduzindo outro profissional, o enfermeiro obstétrico/obstetriz, no atendimento ao parto de baixo risco. Como consequência, as taxas de cesariana caíram à metade do observado nos hospitais privados que seguiam o modelo da conveniência obstétrica, com satisfação das usuárias e redução da internação em UTI neonatal.

A quase ausência de enfermeiros obstétricos/obstetrizes no atendimento ao pré-natal e parto também é outra característica própria do nosso modelo de atenção, embora não exclusiva do país. No Brasil, 93\% dos partos são realizados por médicos, e se considerarmos apenas os partos vaginais este valor atinge para $87 \%$ (dados não publicados da pesquisa Nascer no Brasil). Estudos mostram que midwives adequadamente qualificados têm um papel fundamental na promoção de um processo reprodutivo saudável, prestando um cuidado centrado na mulher e com um manejo racional e adequado dos recursos do sistema de saúde. Uma série do The Lancet denominada Midwifery recomenda esses profissionais como uma solução vital para promover uma atenção de alta qualidade para mães e crianças em todos os países 2 .

O modelo atual, que prioriza o agendamento prévio das cesarianas sem indicação clínica no setor privado, também é composto por outras características: a maioria desses hospitais não tem equipes de plantão e o corpo clínico é constituído predominantemente por médicos externos que possuem consultórios privados onde realizam o acompanhamento pré-natal. Dirigem-se aos hospitais com equipes próprias e externas ao hospital quando necessitam de internações por alguma complicação clínica das gestantes, ou para a realização do parto. Esses aspectos caracterizam também uma prática obstétrica isolada, completamente autônoma, ficando cada médico com a responsabilidade total sobre seus pacientes, sem compartilhamento de experiências e sem contar com uma organização que se responsabilize pelo estabelecimento de diretrizes clínicas, protocolos de atendimento e mesmo oferta de suporte para tomada de decisões.

Uma revisão sistemática 3 sobre intervenções não clínicas para a redução de cesarianas concluiu que há evidências de que se pode reduzir com segurança a prevalência de cesarianas nos casos de mulheres com cesariana anterior, quando se usa uma segunda opinião mandatória e de revisão por pares antes da indicação de cesarianas intraparto, vigilância da prevalência de cesarianas, adoção de estratégias que envolvam análise das práticas de profissionais de 
saúde e sejam baseadas em auditoria e feedback às equipes.

Dessa forma, para a redução das taxas de cesarianas no Brasil, as mudanças que precisam ser implantadas são complexas e em várias frentes. Devem ir para além do suporte financeiro a ser dado pela gestante ao seu obstetra para a criação da disponibilidade de tempo do médico para o acompanhamento do parto vaginal, conforme sugerido no parecer da CFM, muito bem contra argumentado pelos autores desse estudo e inclusive vetado pela ANS por constituir uma ilegalidade.

Foi uma pena que os autores não puderam analisar o pagamento à parte feito pelas mulheres com planos de saúde aos seus médicos, para avaliar o efeito isolado desta variável.

$\mathrm{O}$ artigo de Freitas et al. mostra claramente que ter o mesmo médico no pré-natal e no parto é uma variável mediadora do tipo de parto, mas, como estamos discutindo aqui, não é o fato de ser o mesmo médico em si que representa risco para a ocorrência de uma cesariana. Essa variável é, na verdade, uma proxy do modelo de assistência obstétrica que temos no país, e por isto tem poder para potencializar as associações entre as variáveis independentes desse estudo e a ocorrência de cesariana.

1. Torres JA. Análise da contribuição de um programa perinatal multifacetado para a redução da prevalência de cesarianas em um hospital privado: um subprojeto da pesquisa "Nascer no Brasil" [Tese de Doutorado]. Rio de Janeiro: Escola Nacional de Saúde Pública Sergio Arouca, Fundação Oswaldo Cruz; 2014.

2. Horton R. The power of midwifery. Lancet 2014; 384:1075-6.

3. Chaillet N, Dumont A. Evidence-based strategies for reducing cesarean section rates: a meta-analysis. Birth 2007; 34:53-64.

\section{Os autores respondem}

The authors reply

Los autores responden

http://dx.doi.org/10.1590/0102-311XC0020915

Paulo Fontoura

Freitas, Bianca

Carvalho Moreira,

Andre Luciano

Manoel, Ana Clara de

Albuquerque Botura
As colocações de Maria do Carmo Leal contribuem de maneira importante para o debate e para a busca de soluções para a questão das altas taxas de cesariana em nosso meio. Visto que o espectro de suas colocações transcendem os objetivos do estudo, gostaríamos de enfocar, em especial, duas das questões colocadas, visto serem elas pontos centrais que alicerçam nossas conclusões e que a oportunidade da presente réplica nos permite aprofundar.

Entendemos a surpresa do leitor ao se deparar com um estudo que, aparentemente, busca analisar o impacto de um parecer não referenciado pela Agência Nacional de Saúde Suplementar (ANS). Entretanto, da mesma maneira que a variável "mesmo médico" é uma proxy do modelo de assistência obstétrica no país, conforme detectado, com propriedade, por Leal, entendemos que o teor do parecer defendido pelo Conselho Federal de Medicina (CFM), independentemente de sua aprovação pela ANS, representa o pensamento de grande parte da comunidade obstétrica, fator central que instiga a presente análise.

A opinião do conselheiro federal e coordenador de comissão do CFM, que tratou do tema, ao postular que o incentivo à remuneração é essencial para favorecer a realização de partos normais e que "Esse fator, a remuneração, é sem dúvida um dos elementos essenciais de um conjunto de ações coordenadas que o CFM vem discutindo para reverter a elevada proporção de cesarianas hoje no Brasil" 1, foi na verdade o ponto de partida para o nosso estudo.

Quanto à impossibilidade de acessar com exatidão "pagamento à parte", nossos resultados mostram que a quase totalidade das mulheres que tiveram o parto pelo Sistema Único de Saúde (SUS) com o mesmo médico que realizou o pré-natal declararam não ter pago pelo parto e que a totalidade daquelas que referiram ter pago haviam realizado pré-natal privado deixando em segundo plano, ao nosso ver, a importância da variável "pagamento à parte", tanto como parte do modelo multivariado, por praticamente duplicar a informação da variável "status do parto”, quanto pela construção das conclusões finais. Subsidiando esse raciocínio, os resultados 
informam que o "pagamento à parte" pelo parto não é condição sine-qua-non para ter o mesmo médico, apontando para o "potencial para negociação" paciente/obstetra, já referido por outros estudos.

Gamble et al. 2, em uma ampla revisão de publicações relacionadas aos fatores que circundam decisões pelo tipo de parto, colocam que o contexto psicossocial dos cuidados obstétricos revela um desequilíbrio de poder em favor dos médicos. Os autores concluem que estudos acerca do processo de decisão pelo tipo de parto que não levem em conta o contexto dos cuidados oferecidos e das interações entre mulheres e profissionais devem ser interpretados com cautela.

No que pese a farta publicação de artigos que se ocupam da questão das altas taxas de cesariana no Brasil, permanecem lacunas de informação que permitam entender, de maneira mais ampla, a tão complexa interação de determinantes socioculturais da prática obstétrica, sejam eles institucionais, geográficos, financeiros ou legais, na assim chamada "cultura da cesariana no Brasil”. Uma vez que nenhuma investigação, por si só, será suficiente para responder a todas essas questões, a janela aberta para a discussão, no presente momento, é altamente positiva. Neste sentido, a pertinência e a precisão das colocações de Maria do Carmo Leal, sustentadas por sua familiaridade e por seu profundo conhecimento sobre o assunto, contribuem em muito para a discussão da questão das altas taxas de cesariana, que permanece atual, no Brasil, já há décadas.

1. Conselho Federal de Medicina. Parecer considera ética a remuneração. Medicina Publicação Oficial do Conselho Federal de Medicina 2012; ano XXVII, no 214. http://www.cadastro.abneuro.org/site/jor nal214CFM.pdf.

2. Gamble J, Creedy DK, McCourt C, Weaver J, Beake S. A critique of the literature on women's request for cesarean section. Birth 2007; 34:331-40. 